\title{
SEX HORMONES AND PSYCHOSIS: A REVIEW
}

\author{
M. I. Canelas da Silva ${ }^{1}$, J. Lopes ${ }^{1}$, C. Fernandes Santos ${ }^{2}$ \\ 1 Department of Psychiatry, Hospital Vila Franca de Xira - Vila Franca de Xira, Portugal \\ 2 Department of Psychiatry and Mental Health, Hospital Garcia de Orta - Almada, Portugal
}

\section{OBJECTIVES}

The aim of this work is to shortly review current knowledge on the topic of sex hormones in psychotic disorders.

\section{BACKGROUND}

Recent studies have shown that sex hormones, such as estrogens, progesterone, testosterone, dehydroepiandrosterone sulfate (DHEA-S) and prolactin, play an important role in psychotic disorders like schizophrenia.

These hormones seem to be related to the pathophysiology, course and severity of disease, symptomatology and gender differences in psychosis ${ }^{1-9}$.

\section{MATERIALS AND METHODS}

A literature search in the PubMed database was conducted, using the search terms "sex hormones", "sex steroids", "psychosis", "psychotic disorders" and "schizophrenia". Relevant articles were also sought from citations.

A non-systematic review was performed, using articles published up to December 2018. Nine relevant articles were selected.

\section{RESULTS}

Most of the available studies focus on the role of sex hormones in schizophrenia and first-episode psychosis (FEP).

\section{- Estrogen hormones are the best studied ${ }^{1,3,7,8}$.}

Research over the last two decades has established for them a clear neuromodulatory role in the pathogenesis of schizophrenia.

Estrogens seem to have neuroprotective properties, protecting the brain from the development and severity of mental disorders like psychosis. On the other hand, states of estrogen deficiency have been frequently reported in women with schizophrenia.

The "estrogen hypothesis" can be an explanation to gender differences in schizophrenia, such as higher incidence in men, older age of onset and less severe course of illness in women. It is also consistent with the existence of a peak of onset in females after the age of 40 , which may be associated with the hormonal decline in menopause.

Clinical studies have shown that women with psychotic disorders demonstrate increased symptom severity, greater relapse rates and more hospital admissions during phases of low circulating sex hormones: perimenstrual, postpartum and postmenopause.
An increasing amount of literature has explored estrogen therapy as a potential adjunct to antipsychotics, as it might improve cognition and positive symptoms, but this remains controversial.

\section{- Progesterone 1,2,9}

The impact of this hormone has been far less studied.

Existing data suggest that it is a key modulator of central systems implicated in schizophrenia, such as the dopaminergic system. There is some evidence of dysregulation of progesterone levels in patients with schizophrenia, particularly in women during the mid-lutheal phase of menstrual cycle.

It can also contribute to gender differences in psychosis. Better prognosis and response to medication in women may be related to the combinated effects of estrogen and progesterone on functions like cognition and sensorimotor performance.

- Prolactin is commonly increased in psychotic patients, even in those who are antipsychotic-naïve or with an at-risk mental state. The "stress-prolactin-dopamine hypothesis" has been proposed to explain how it acts in the transition to psychosis. Since it suppresses the production of the gonadal estrogens and progesterone, the hyperprolactinemia could explain those hormonal deficiencies ${ }^{7}$.

- Testosterone and DHEA-S importantly influence dopaminergic, glutamatergic and GABAergic systems, which act in the pathophysiology of psychotic disorders ${ }^{2,4,5,6}$.

It has also been reported that their levels may impact psychopatological manifestations of schizophrenia. Specifically, many studies revealed a negative correlation between the levels of testosterone and the severity of negative symptoms or cognitive deficits.

A recent review and meta-analysis ${ }^{5}$ has found significantly higher levels of DHEA-S in psychotic patients compared to controls, the difference being more significant in men. This might be perceived as a stress response mechanism, since it is produced after the release of $\mathrm{ACTH}$, or related to increased dopaminergic transmission in acute psychosis. Levels of testosterone differ in FEP (increased free and unaltered total testosterone) and acute relapses (the opposite pattern).

\section{CONCLUSIONS}

There is still much to be discovered on the present topic. Future research is needed, as improved understanding of the role of sex hormones in psychosis and their potential application in treatment would be of significant clinical value.

REFERENCES: 1. Bulut S, Güriz O; 2016. The relationship between sex hormones profiles and symptoms of schizophrenia in men. Comprehensive Psychiatry; 89:186-192. 2. Buoli M, Caldiroli A et al; 2016. Sex Steroids and Major Psychosis: Which Role for DHEA-S and Progesterone? Neuropsychobiology; 73:178-183. 3. Gogos A, Sbisa A et al; 2015. A Role for Estrogens in Schizophrenia. Clinical and Preclinical Findings. International Journal of Endocrinology; 2015:615356. 4. Huber T, Tettenborn C et al; 2005.Sex hormones in psychotic men. Psychoneuroendocrinology; 30: 111-114. 5. Misiak B et al; 2018. Testosterone, DHEA and DHEA-S in patients with schizophrenia: a systematic review and meta-analysis. Psychoneuroendocrinology; 89: 92-102. 6. Moore $L$ et al; 2013. Serum testosterone levels are related to cognitive function in men with schizophrenia. Psychoneuroendocrinology; $38: 1717-1728.7$. Riecher-Rössler A; 2016. Oestrogens, prolactin, hypothalamic-pituitary-gonadal axis, and schizophrenic psychoses. Lancet Psychiatry; November 14, 2016. 8. Silva T, Ravindran A; 2015. Contribution of sex hormones to gender differences in schizophrenia: A review. Asian Journal of Psychiatry; 2015. 9. Sun J et al; 2016. Progesterone: The neglected hormone in schizophrenia? A focus on progesterone-dopamine interactions. Psychoneuroendocrinology; 74:126-140.

mariainescs00@gmail.com 